\title{
DE CÓMO LAS TICS PODRÍAN COLABORAR EN LA INNOVACIÓN SOCIO-TECNOLÓGICO-EDUCATIVA EN LA FORMACIÓN SUPERIOR Y UNIVERSITARIA PRESENCIAL
}

\author{
(HOW ICT COULD COOPERATE TOWARD THE SOCIO- TECHNOLOGICAL INNOVATION IN FACE \\ TO FACE UNIVERSITY EDUCATION)
}

\author{
Beatriz Fainholc \\ UNLP-CEDIPROE (Argentina)
}

\section{RESUMEN}

La gran presión de las TICs atraviesan el entramado de la realidad socio-económica y políticocultural de la sociedad de la información y penetran en el campo formativo de personas y grupos y en especial, universitario presencial, no sin resistencias y dilemas. La innovación tecnológico-educativa en esta formación significa otorgar nuevas tramas de sentido a la interacción pedagógica cotidiana.

Las TICs configuran "ambientes de aprendizaje" que flexibilizan la educación tradicional presencial pero no son panaceas. Exigen desmistificar y reformular concepciones y prácticas curriculares universitarias y revisar la formación del profesorado hacia el desarrollo de competencias complejas que acompañen reflexivamente estos procesos. El dominio para acceder, manejar, integrar, distribuir, evaluar, producir y recrear información, es central para transformarla en saber al tiempo que reconstruir y articular estos procesos y resultados virtuales, de modo crítico y contrastado en la realidad concreta, es una necesidad en la enseñanza universitaria presencial. Flexibilizar actitudes y propuestas ayuda a funcionar con efectividad y pertinencia formativas sustantivas.

Palabras clave: innovación socio-tecnológica, incorporación de TICs en currículo, formación flexible universitaria, nuevas tramas de sentido, formación conceptual y metodológica del profesorado.

\footnotetext{
ABSTRACT

The ICT high pressure in daily days of the socio- economic and politic- cultural of the information and knowledge society penetrates at every formative area of people and groups, specially at face to face university education level, not without presenting reluctances and dilemas.
} 
The technological innovations in education means meanly, to give another sense to the everyday pedagogic interaction. ICT build "learning environments" which should to flexibilize face to face tradicional education but as everbody knows, they are not panaceas. To desmistify and reformulate university conceptions and practices within the currículum and the training of faculty, could be done with the help of ICT in order to develop complex competences and reflective processes. To achieve expertise in order to access , manage, integrate, distribuite, evaluate, produce and recreate information is very important in university teaching to transform it and at the same time, to rebuild and articulate ICT in a mix of virtual and conventional educational processes and results.

To flexibilize attittutes and proposals incorporating ICT to the university curriculum and organization will help to renovate educational practices towards substantial and effective changes with formative pertinence.

Key words: socio-technological innovation, ICT curricula articulation, flexible university education, faculty training in new conceptions, methods and competences, critical approaches.

\section{CONTEXTUALIZACIÓN}

Hagamos un ejercicio de anticipación. Pensemos que nuestra sociedad ha logrado el transito a otra instancia civilizatoria donde sus fuerzas poderosas socioculturales constituyen el conjunto de conceptos y practicas referidas a un desarrollo humano sostenible, contribuidoras a un poder asociativo global. Ello se halla posibilitado por las redes electrónicas y el surgimiento de los medios de comunicación horizontalparticipativos apoyados en las TICs (web 2.0 y otros).

Sin embargo y a pesar de los logros, los escenarios de esta sociedad del conocimiento continúan demostrando ser ambiguos e inciertos: requieren propuestas organizacionales con personas de esquemas representacionales flexibles para enfrentar incertezas y generar respuestas imprevisibles. Se ha entendido que la energía central es el saber y las competencias requeridas para un "saber y querer hacer" que favorezcan producirlo. Sin embargo, aún existen dificultades en el logro de un desarrollo autosostenido del conocimiento. Los pilares de esta sociedad que se sostienen en el poder de la gente y sus instituciones (por sus saberes, valores y actitudes), en el poder del aprendizaje, de la productividad y de la integración sociocultural global, deben continuar en la profundización de logros auto sustentable, o sea duraderos a lo largo del tiempo, con capacidades centrales que las personas y los grupos deberían poseer, demostrar y aplicar.

Enormes esfuerzos de anticipación e imaginación respecto de las características de este estadio social deben ser tomadas en cuenta para la satisfacción equitativa de 
sus necesidades, -que conlleva a la concreción de la e-inclusión-, antes que abocarse a una introducción caprichosa de innovaciones, porque se ha pensado en el valor social de la tecnología antes que en su consumo desenfrenado. Es decir que ya es materia conocida y reconocida el porque y para que de los medios y mediadores tecnológicos, lo que es lo mismo que decir: que la persona a pesar de las transformaciones sociales satisface primero su necesidad comunicativa y expresiva: lo que no es lo mismo que tener el ultimo modelo del celular que toma fotos, emite música y adivina el número telefónico a marcar.

Se ha apuntado así a un proyecto que sostiene una "alternativa sociedad del conocimiento", que afirma el valor y condición de la autonomía como estado personal y colectivo para crear sus propias instituciones y ser capaz de revisarlas, apropiarlas y transformarlas (Castoriadis, 2006) ${ }^{1}$ continua y autoevaluadamente.

Si bien se han logrado ciertos grados de reconocimiento de la identidad personal, organizacional y regional de países en el ciberespacio inter/ transnacional, dados por el uso participativo, horizontal y comunicativo de las TICs (web 2.0 y otros), se continua aún, trabajando arduamente para reconocer nuevos patrones flexibles para producir ideas e insights diversos, ya que la innovación continua siendo imprevisible y permanente.

Por ello continuar en la creación de nuevo saber para que las mentes se abran, se comuniquen, dialoguen pública y conectadamente, a partir del espacio cultural de una internacionalización ciberespacial de Internet, si bien logradas, sigue requiriendo explotar mas las potencialidades enormes de las TICs y las redes de modo creativo. Esto es saber alejarse del pensamiento convencional, además de superar la racionalidad instrumental o artefactual que caracteriza el consumo desenfrenado y final de equipos.

Creeríamos que de este modo, se podría producir el tránsito a otro tipo de sociedad de la información y del conocimiento, que implica personas, grupos y organizaciones que reconocen que educarse es entre otras características, aprender la mayor cantidad de lenguajes y formas de presentar/representar el conocimiento, para lo cual ha sido necesario otorgar otro sentido a la tecnología y apropiarse pertinentemente de las TICs.

Entonces para equipar personas que enfrenten la sociedad de la información (S.I.) y la economía del conocimiento, entre las innumerables tareas, la universidad debe revisar su modus operandi, su modelo organizativo y cómo gerenciar (o sea, 
su management) su propuesta de educación superior conjuntamente a su modelo pedagógico. Todo ello inscripto en los contextos socioculturales referidos, aplicados a la educación, y en especial, a proyectos universitarios con modalidades sociotecnológico-educativas que deben ser no convencionales, lo mas flexibles para satisfacer las cambiantes demandas formativas que la historia nos sorprende.

Se ha reconocido que el sentido de la misión formativa de una sociedad se instituye e inscribe dentro de un marco y tiempo histórico-socio-cultural específico. Para ello asimismo, se ha requerido de instituciones que se revisen y anticipen la formación de ciudadanos/as lúcidos, habilidosos y solidarios para favorecer inclusiones socioculturales diversas. Demostrado ya fue que articularando las TICs con relevancia y efectividad al interior de las mediaciones tecnológicas, parte de la cuestión se logra. Se esta progresando, de este modo en democratizar con otro sentido la producción y distribución de información que se convierte en válida y valiosa para robustecer las potencialidades de una ciudadanía emergente, según las nuevas demandas de estos tiempos.

\section{LA PREGUNTA}

Entonces la pregunta, -al identificar el marco donde inscribir dichos esfuerzos socioculturales específicos personales y colectivos-, es ¿cómo hacerlo?, ¿cómo se hace?, ¿cómo han hecho los que lo han hecho? y/o ¿cómo continuar en una línea de mejoramiento? ¿Cómo extraer reflexiva y aplicativamente conceptos y prácticas (a modo de una propuesta de "ingeniera reversa") con sentido crítico y poder horizontal, para transferirlo y proceder en otras situaciones? Es decir, rescatar los procesos por los cuales se ha sabido apropiar las TICs como colaboradoras de innovaciones socio-tecnológicas adecuadas y como se las ha podido articular en la cultura y en las propuestas formativas. Cómo el sistema universitario haría para superar la crisis en la que está inmerso, siendo capaz (no sin retrocesos) de enfrentar la presión socio-política y económico-cultural local y global, y responder a las necesidades emergentes de una economía intensiva en conocimiento. El interrogante es qué pasos y previsiones son urgentes reinventar para una universidad de resultados sostenibles y auto-sostenidos, más efectivos en costo, equitativa para un acceso ampliado, respetuosa de las diferencias personales y culturales y, democrática o inclusiva para brindar más y mejores oportunidades a los estudiantes si se lo compara con las universidades convencionales conocidas. 
Entonces pues, se trata de zambullirse en los procesos que algunas o varias han sabido realizar u otras habrían de reestructurar en tales centros educativos, formar a su dirigencia y a los profesores/as universitarios, al tiempo que transformar las situaciones de enseñanza y aprendizaje para que las personas desarrollen saberes y actuaciones "respondentes" (Stake, 1976) ${ }^{2}$ a la nueva visión societal, con actitudes proactivas y comprometidas, flexibles y transformativas.

Si esta descripción responde al tiempo histórico-cultural que se vive y se vivirá, se necesitan decisiones enérgicas y sostenidas para imaginar y liderar transformaciones, negociar contradicciones y conflictos inherentes a todo contexto, justipreciar situadamente los pros y contras de las tecnologías, a partir de una óptica alternativa para remover anacronismos que resisten revisiones profundas.

En consecuencia, las innovaciones socio-tecnológicas genuinas y pertinentes que la educación superior necesita, se aproximarían progresivamente a flexibilizar las instancias de formación "cara a cara" porque reformula su ideario principista y entre otros elementos centrales, reconoce el aporte del modelo articulador que proponen las TICs con sus marcos de trabajo de aprendizaje y enseñanza electrónicas, y con ello, inauguran nuevos ambientes socioeducativos. La contribución sistémicoholista consecuencia de estas propuestas, estaría dada por el logro de una formación flexible y abierta de personas concebidas como tendientes a constituirse en analistas simbólicos, experimentadores y anticipadores de problemas que proponen soluciones alternativas para sus sistemas socioculturales.

\section{INNOVACIÓN SOCIO-TECNOLÓGICA EN LA ORGANIZACIÓN UNIVERSITARIA}

La sociedad de la información demostró y continúa demostrando estar movida por la energía que representa la producción de saber o conocimiento ("building knowledge powered society"), resultado de la energía sinérgica de personas, grupos y organizaciones. Su poder radica en el aprendizaje, la productividad, la integración socio-cultural y la formación de una nueva ciudadanía ${ }^{3}$. Entonces fortalecer las habilidades de predicción de escenarios futuros durante la formación universitaria, -para contraponerla al aun vigente pensamiento convencional lineal-, apelar a una perspectiva holista, inter/transdisciplinaria y ética que elabora, comparte, configura, contribuye y distribuye saber, conduce a pensar que si dicha organización universitaria no está movida por el poder del conocimiento socialmente útil, es y será obsoleta. 
Una innovación socio-tecnológica supone producir un quiebre respecto de lo que es acostumbrado o rutinario en una organización social para superar la generación repetitiva o mecánica de concebir y producir los procesos y resultados requeridos, en nuestro caso, formativos. Urge o se necesitan entonces, productos nuevos porque los existentes ya no satisfacen las necesidades de la realidad, los procesos que se viven se vaciaron de sentido para los usuarios y los resultados que se obtienen no se vinculan con las demandas histórico-culturales contextuales del trabajo y de la vida de relación en general.

Correlativamente a ello también, se entiende hoy la "gestión del cambio educativo" como el gerenciamiento de un diseño propuesto para una innovación socio-formativa mediada por tecnología, de preferencia con TICs, que las vincula de modo sustantivo con la cultura a través de la enseñanza, orientada por equipos que aporta su experticia para alcanzar tales fines. Claro que ello, hoy debería asegurar la elección de concepciones reflexivas superadoras de anacronismos y el uso de metodologías y recursos en forma, contenido y tiempo del modo mas adecuado a una propuesta viable, factible y pertinente si se trata de producir genuina y sostenidamente resultados a su vez, sean tangibles y mensurables, trasferibles y trascendentes.

\section{Fuentes de resistencia al cambio}

Sin embargo, el cambio no tiene gran aceptación: personas y organizaciones aún y a pesar de los logros, se resisten. Entre las fuentes individuales de resistencia al cambio se hallan los hábitos adquiridos y convertidos en rutinas que no facilitan sino anquilosan, la realización reflexionada de actividades con significado. La resistencia organizacional apunta a diversas inercias estructurales de mecanismos construidos ancestralmente que si bien producen estabilidad, son anacrónicos a las demandas de tiempos culturales diferentes. La inercia de una institución o grupo se da porque sus normas actúan restringiendo el cambio, sin enfrentarlo y repensarlo por lo que también, contribuyen a la resistencia nombrada.

Todo cambio tecnológico de este modo, apuntaría a no cesar de profundizar la calidad de sus procesos, productos y servicios en su generación y gerenciamiento, dentro de una (tradicional) organización universitaria, para nuestro caso. Lo que se vincula a la integración de subprocesos que demuestren ser en la práctica, oportunos y pertinentes porque son útiles, valiosos, viables, transparentes, amistosos, precisos, realistas, prudentes y éticos para las personas, la sociedad y la cultura. 
Se apunta así a una (alternativa) incorporación inteligente y criteriosa - lúcida y no consumística- de las TICs apoyadas en valores que sostengan los esfuerzos personales y grupales para un desarrollo sociocultural, científico-tecnológico y productivo visible y sostenido. Se relaciona en su ideario y en la práctica con la flexibilidad de las propuestas formativas porque reina una amplia confianza, autenticidad y apertura. También un fomento al protagonismo de los usuarios (estudiantes, profesores y administradores/as) como responsables y partes interesadas en un aprendizaje organizacional para una maduración y mejoramiento -personal y social- progresivos. Asimismo se trata de un apoyo a la participación porque todos y todas, son parte comprometidos del cambio cultural y de mentalidades ya en articulación con las coordenadas factuales y virtuales.

Estas innovaciones reconocen para su construcción, que su energía pertenece a todos y todas: está conformada por la e-inclusión (al desarrollar saberes y competencias vinculadas a las TICs), hacia un empoderamiento de los usuarios, en dirección a aprendizajes sustentables a lo largo del tiempo y durante toda la vida. También, apuntan a organizaciones educativas universitarias de amplio acceso y flexible formación que propongan programas (de enseñanza, investigación y prospectiva) de calidad y pertinencia, -dentro de una internacionalización creciente y sin perder la identidad local-, de una formación clave para anticipar modelos educativos de avanzada.

Entonces, en estas propuestas innovadoras curriculares de las instituciones universitarias, el contenido disciplinar e interdisciplinar y las formas mediadas por tecnologías, aparecen con marcas ciberculturales. Vivir la interdependencia con los mundos sintéticos (por solo nombrar el ejemplo de "Second life"4) implica auténticas transformaciones en donde no bastan las máquinas y las simulaciones sino se toman en cuenta en la construcción de la subjetividad, lo:

- subjetivo: con una remoción/cambio de teorías implícitas o concepciones erróneas existentes en personas y grupos desde las que se interpreta ahora el mundo y la vida con tecnología en general, y

- objetivo: por la práctica contrastada del objeto de la transformación, al analizar su intencionalidad manifiesta e implícita, su contenido, estrategias metodológicas, su evaluación e impacto lateral o riesgoso posible.

Este sería el ambiente de acceso e intención ampliadas, donde más personas construyen y comparten el saber, interactúan con la incertidumbre y las 
contradicciones en escenarios nuevos de comunicaciones híbridas horizontales, con una promoción de actitudes mas pro-activas, anticipatorias y monitoreadas para una expansión insospechada. Las TICs no siendo panaceas han demostrado poder coadyuvar a estas situaciones.

Por lo tanto la selección, combinación y aplicación de tecnologías electrónicas y de mundos sintéticos, debería inscribirse en una estrategia más flexible y comprehensiva de la enseñanza para inventar los aprendizajes mediados, ahora electrónicamente.

Estas transformaciones que las universidades no pueden soslayar como usinas productoras de saber (o de verdad como se decía en otros tiempos), profundizan su razón de existir, -dentro de unidades socioculturales mayores de comprensión sistémico-holista-, al producir quiebres, amplificar las funciones superiores de pensamiento individual y organizacional y al aplicar de modo contrastado en campo, procesos y productos reconstruidos o imaginarios que parten de sofisticados insumos en constante remoción.

Se está bastante lejos de satisfacer estas necesidades a pocos años de cumplirse el 1er decenio del siglo XXI.

\section{LAS TICS SE INCORPORAN EN EL CURRÍCULO UNIVERSITARIO PRESENCIAL PARA FLEXIBILIZARLO}

Entonces el desafío en una sociedad superadora de una concepción del conocimiento elitista y acumulativo (además de hegemónico), requiere revisitar varios componentes: no sólo de acceso y uso equitativos de las TICs para no quedar/ dejar excluidos/as socialmente a personas, sino por su apropiación conceptual y metodológica con una transferencia aplicativa y contrastada a otras instancias organizacionales y culturales. Anticipar y recrear respuestas para una inserción articulada de las TICs en los programas educativos superiores de formación, particularmente, de profesores/as es clave ${ }^{5}$.

¿Cómo? Rescatar, ensayar e imaginar prácticas socioeducativas con formas valiosas de producción de conocimiento socialmente útil a partir de las nuevas coordenadas virtuales en una aplicación innovadora con propuestas de elaboración de síntesis, puede ser un camino. 
Se debe tener presente que si bien las TICs y sus mundos sintéticos se constituyen por códigos simbólicos que demostraron que no son panaceicos, son los vigentes en la sociedad actual de la información, y por ende, urge capitalizarlos además de investigarlos de modo transversal en todos los campos del saber.

El tema no es simple: aún reinan rigideces y "pensamientos únicos", donde muchas de las innovaciones como fuertes iniciativas de avanzada, tienen gran dificultad de generalización porque no pueden ser asumidas por la organización universitaria como un proyecto comprehensivo o global que incorpora de modo crítico y recreador a las TICs.

Se requiere un gran compromiso institucional en valores y espíritu representativo de variados idearios para configurar auténticamente una cultura universitaria flexible que promueve la producción y uso social y equitativo del saber, la investigación científico-tecnológica y la transferencia práctica e inclusiva a la sociedad, por una apropiación pertinente de las TICs para que realmente sirvan de un modo crítico. No aceptar transferencias en bloque de tecnología sino que cada una sería analizada en su propia especificidad y consecuencias, teniendo en cuenta sus debilidades y fortalezas dentro de un proyecto alternativo.

Se retoman los pensamientos de Durkheim (1963) que sostiene que una institución escolar -en nuestro caso una organización educativa universitaria y/ o de nivel superior-, es el espacio y el lugar de socialización e integración sociales que interactúa con la sociedad y la cultura en mediaciones diversas, para producir saber y consolidarse en el tiempo, se añade el presupuesto central de propuestas de renovación y crítica, dentro del paraguas de la inclusión social. Porque escenarios y actores, tiempos y lugares actuales, pasados y expectativas futuras, son diferentes: transcurren en ambientes cada vez más virtuales y desformalizados como precarios e inciertos, y así demandan otras propuestas y soluciones.

Generar, multiplicar y profundizar saber tecnológico (Fainholc, 2001) ${ }^{6}$ de modo distribuido y compartido, contribuye a la discusión crítica e integración sociocultural transformativa en épocas donde la tecnología penetra toda la realidad pero produce al mismo tiempo, fragmentación y disloque.

Asimismo existen diferentes razones para utilizar la tecnología y en especial las TICs en la enseñanza superior (Bates, 2001) ${ }^{7}$. Se trata de una serie de factores que han llevado a las organizaciones universitarias a incorporarlas en la enseñanza, el aprendizaje, la extensión y la investigación. Algunas con la modalidad virtual en 
una implementación simultánea y articulada, conducen a comprender porque y cómo las TICs podrían colaborar en la innovación socio-tecnológico-educativa en su integración en la formación superior y universitaria presencial.

En general se piensa que se utiliza la tecnología y las TICs, porque existen de modo objetivo o para no quedar rezagados en la "moda"; otras depende de las personas y su posibilidad de decisión según el cargo que ocupen (por la cuota de poder y/o profesionalismo que posean), otras veces se trata de líderes innovadores en general incomprendidos que articulan las TICs en proyectos pioneros y sin apoyo alguno. Sin embargo en todos los casos, se podría sostener que:

- Mejoran la calidad del aprendizaje porque de este modo es más actualizado, variado y rico al llegar de modo distribuido.

- Ofrecen a los alumnos el aprendizaje y la práctica de las habilidades cotidianas de interacción y comunicación con una tecnología de la información cada vez más común y que existe en la realidad cotidiana y se necesita en el trabajo y en la vida.

- Amplían el acceso a la educación, con programas no sólo de formación sino de extensión a la comunidad, aumentando la retención de los estudiantes remotos con el seguimiento personalizado en la formación y otros.

- Posibilitan la apropiación de códigos simbólicos convergentes al interior de la sociedad tecnológica actual.

- Permiten desarrollar más autocontrol ejecutivo no sólo de rutinas simples para aprender sino de desarrollar metaconciencia de los procesos ejecutivos subyacentes que conjuntan las personas y las máquinas.

- Reducen -como algunos sostienen-, los costos de la enseñanza, lo que habría que verificar en términos del contexto socio-económico de país o región e institución universitaria específica, que las incorpora y para que fines, lo que significa.

- Mejorar la relación entre costos y efectividad de la enseñanza.

- Coadyuva a investigar mundos imprevisibles en términos concretos.

La educación como dimensión sociocultural y productiva que se visualiza en la universidad, así debe evidenciar un lugar de alta relevancia en la elaboración y aplicación de conocimiento científico-tecnológico socialmente útil, con valores potentes de apertura y democratización subyacentes. Ello exige reorientaciones urgentes en base a los cambios acelerados que con la aparición de los nuevos códigos 
tecnológicos se dan en las mediaciones y que impactan en las relaciones sociales en general.

Las mismas se deberían insertar en marcos de reorganización de la cultura y de la producción y reclaman un papel estratégico a cumplir en la currícula de educación superior -en concepción y metodologías-, con calidad y equidad en sus procesos.

Parecería que incluir y/o centrar la formación en mediaciones electrónicas resulta interesante, situado, innovador y anticipador desde el punto de vista formativo si bien no presenta de ningún modo, resultados taxativos y debería ser materia de discusión e investigación constantes debido a lo no poco que se debe profundizar acerca de las potencialidades enormes de las TICs, Internet y los mundos sintéticos, en los proyectos educativos.

Pero al mismo tiempo, se tiene la sensación que sin estas mediaciones, poco y nada se contribuye a formar personas y grupos "plantados" socio-culturalmente hoy para construir lo local, dentro del concierto social internacional. O lo que es lo mismo, desde el punto de vista de la crítica, al apropiar localmente tecnología. Que es lo que deberían hacer los "dominados" adaptando componentes materiales y simbólicos "dominantes", en términos tecnológicos, a fin de poder revertir una situación de inequidad, muchas veces histórica.

\section{La innovación socio-tecnológica de la educación presencial universitaria}

Si pensamos en cómo las TICs podrían colaborar en la innovación sociotecnológica de la educación presencial convencional universitaria, no entendida para convertirse necesariamente en una transición forzosa a una universidad virtual del e-learning (con todas las críticas y fracasos que se han registrado y los ajustes evaluativos que se recomiendan realizar para poder mejorar esfuerzos), los nuevos escenarios que innovan y flexibilizan con la articulación de las TICs en la presencialidad, cabría ser del siguiente modo posible.

\section{Como:}

- Formas de representar, codificar, almacenar, distribuir, comunicar e interactuar con el saber disciplinario cercano y remoto, lo que exige reconceptualizar sentidos, tiempos y espacios de las mediaciones y mediadores: entre ellos, desde el rol tradicional de la lectura lineal del libro impreso y la palabra 
como medio comunicacional privilegiado para pasar a entornos hipermedias electrónicos virtuales con la red Internet en diferentes articulaciones textuales y sociales. También, pensar en provocar nuevas configuraciones de significado dentro de las perspectivas macro histórico sociales, culturales, económicas y comunicacionales "líquidas" (Bauman, 2004) .

- Reconocer la horizontalización de oportunidades de aprender en sistemas flexibles, o existentes "mas allá de la escuela" -en cualquier nivel y modalidad formativa-, al valorizar lo no formalizado y abierto, posibilitado por la interacción con las tecnologías. Reconocer que las mismas también despiertan contradicciones y resistencias como adhesiones irreflexivas por supuestas adopciones panaceicas, -que habrá que desmistificar-, dadas por un consumo marketinero que presionan al currículum de la educación formal y no formal.

- Rescatar, dentro del contexto de la diversidad cultural, -aunque no siempre de equidad social-, la configuración de saberes a partir de mediaciones y mediadores de navegación en Internet realizados en cibercafes, teléfonos móviles conectados en juegos grupales, mensajerías de texto, producción de videos You-tube o audios de podcasting. Los mismos si bien requieren ser validados y evaluados/ investigados, deberían ser apropiados ya que es parte del paisaje de los espacios de la vida pública y privada. De este modo se alteran, desarrollan y jerarquizan competencias socio-tecnológicas en los estudiantes que en general, están disociadas de lo académico formal universitario.

- Exprimir las posibilidades que las TICs multiplican, replantean y aceleran en la interacción socio-formativa a través de la interactividad tecnológico-educativa (como a través de la web.2) ${ }^{9}$ para superar desterritorializaciones transnacionales y producir compromisos de trabajo serios por transferencia social a las comunidades para una aterrizada satisfacción de necesidades y resolución de problemas. Es decir la universidad a través de sus "Servicios de extensión universitaria" propicia y se corresponde con programas de formación que aprovechen y potencien la productividad por un aprovechamiento pedagógico de la transversalidad socio-virtual de las TICs.

- Investigar al interior de las propuestas curriculares y en la acción, la transversalidad de las TICs en proyectos sinérgicos de variadas disciplinas o áreas del conocimiento, para replantear y potenciar la construcción del saber y el hacer de modo anticipado, estratégico y sostenido. 
- Robustecer la identidad organizacional, nacional y personal en el ciberespacio inter/transnacional por la práctica del respeto a lo diferente en situaciones comunicativas interculturales mediadas por tecnología y al exprimir nuevas posibilidades. Al realizarse por introspección (por ejemplo con el uso de weblogs) y por confrontación de ideas (por ejemplo por el uso de las redes colaborativas), para la producción de saber tecnológico nuevo (por ejemplo a través de webquests al aprovechar cualquier contenido disciplinario o de áreas de conocimiento), o al profundizar la metacognición cuando el estudiante produce insights por situaciones creadas para que "la mente se abra", o cuando se comunica y dialoga pública y conectadamente con otros en el espacio acústico y cultural de Internet.

- Ensayar modelos electrónicos híbridos de educación o blended learning ${ }^{10}$ que resultan en un mix de la enseñanza "cara a cara" con la educación virtual soportada en sistemas (plataformas) tecnológicos de administración de contenidos y orientación tutorial. En estas propuestas es clave la justa selección, combinación, aplicación y seguimiento de cada una de las tecnologías y de las TICs que se ha decidido articular en el diseño instruccional de una unidad o clase, y/o curricular de un curso o de una carrera.

- Lo que la creatividad de los administradores y del profesorado haga brotar y lleve a experimentar más lo que los estudiantes traigan a clase o sugieran como temas y actividades, el camino está abierto.

De este modo, la educación superior con la incorporación de TICs profundizaría lo que ya está demostrando como relevante en cuatro tipos de cambios:

- En el profesor: en su rol organizacional, administrativo y técnico, social, psicológico y cognitivo.

- En el estudiante que debe aprender a usar con inteligencia y exprimir las TICs, para su selección, evaluación, organización y aplicación de la información.

- En la concepción y metodologías de la enseñanza con redes y situaciones participativas que más que nuevas metodologías con TICs implican nuevas perspectivas para entender la realidad formativa mediada en aras de tratar de enriquecer y mejorar la práctica educativa de modo reflexivo. 
- Administrativo-organizacionales al reconocer el marco sociocultural del ciberespacio en estrecha interdependencia con los vínculos formativos "cara a cara”, establecidos por los cambios históricos- culturales y socio-emocionales.

\section{El protagonismo de la formación flexible}

Entonces profundizar las demandas que la sociedad de la información en su tránsito a una sociedad mas pertinente del conocimiento, -preocupada por sostener una línea de cambio socio-tecnológico sustentable-, propone situaciones que provoquen enfrentar, dilucidar, reflexionar y aplicar tecnología del modo mas flexible. Considerarlas así como organizadoras de "ambientes de aprendizaje", significa revisar los componentes centrales del currículum y de los contextos instruccionales o de enseñanza para formar a personas y grupos dentro de transformaciones muy profundas en las concepciones y roles socioeducativos presentes, con otro impacto futuro.

Las propuestas educativas electrónicas mediadas por las TICs además de implicar entornos virtuales de aprendizaje en general en forma de cursos, seminarios en línea y/o trabajos de investigación en grupos colaborativos, supone entender que el acceso, la construcción, distribución y aplicación/uso del conocimiento procesado puede darse en situaciones combinadas o mixtas con la educación presencial. Ello significa revisar diseños curriculares e instruccionales desde la perspectiva de una Tecnología Educativa Apropiada y Crítica ${ }^{11}$.

Toda innovación propone un programa formativo anticipador para la formación de sus protagonistas requiere y se nutre de recursos materiales y simbólicos que les permitan soportar el costo de instituirlas al absorber/reajustar las debilidades y fracasos como los errores inevitables en cualquier implementación nueva. De ahí que impulsar una comunicación fluida entre las unidades y diversos sectores de la universidad (administradores, departamentos, carreras, profesores/as, técnicos y otros), a fin de convocarlos para la renovación reflexiva, es una necesidad constante a fin de fortalecer la nueva (ciber) cultura general y en especial, educativa.

Hoy configurar una "organización que aprende"12 de modo flexible, aterrizado, crítico y abierto, de nivel universitario debería combinar e innovar su presencialidad característica con ambientes virtuales, dentro de una aplicación en contextos concretos y con objetivos precisos, de procesos nuevos. Ello desafía a cualquier organización convencional a establecer un proceso de adaptación y apropiación no 
adopción, que cambia y se reformula a la luz de los diversos requerimientos epocales y socioculturales, para producir logros sostenidos a lo largo del tiempo.

La innovación de flexibilizar la educación presencial articulando las TICs supone revisar además de la esfera administrativo-organizacional, la pedagógica mediacional comunicativa, que investigue su incidencia directa en sus determinaciones formativas.

La variable tecnológica como la semiológica acentúan lo educativo porque mediaciones y mediadores coadyuvan en la especificación del logro (o no) de las metas de aprendizajes (según estilos cognitivos e inteligencias múltiples mediante) de los estudiantes. Además considerando los contenidos disciplinares y su secuencia en la enseñanza, la combinación de estrategias metodológicas seleccionadas por el tutor/ profesor/a, el uso adecuado y flexible de todos los recursos integrados para lograr entre variados imponderables de la práctica, la efectividad pedagógica en contextos formativos superiores.

En consecuencia, el cambio socio tecnológico en la universidad convencional que ha movilizado sus estructuras y articula concepciones y procedimientos relacionados con las tecnologías electrónicas, ha necesitado y sigue requiriendo de ciertos prerrequisitos tales como:

- Poseer una visión compartida en la que todos acuerden.

- Desechar formas viejas de pensar caracterizadas por rutinas estandarizadas para solucionar problemas o cumplimentar tareas.

- Considerar que los procesos formativos, deben explicitar/deconstruir concepciones ocultas para el diseño curricular e instruccional, al tiempo que garantizar insumos administrativo-organizacionalesy degestión, de mediaciones tecnológicas para la comunicación en conectividad, incluyendo las funciones de evaluación e investigación que se hallan en interacción interdependiente dentro de una cultura en la acción.

- Fortalecer una gran y abierta comunicación entre todos y todas es insoslayable, más allá de los niveles diferenciados, con confianza y sin temores, para permitir el desarrollo de la autoestima y autonomía organizacional y académica, si se persigue la sostenibilidad. 
- Fertilizar a través de la investigación en la y para la acción, toda interacción y comunicación mediadas con las TICs.

- Supeditar el interés personal y los intereses departamentales fragmentados por el interés en el trabajo en conjunto de la universidad u organización de nivel superior, carrera o departamento que se trate, a fin de alcanzar la visión compartida y productiva de la misma en su impacto mayor.

- Fomentar compromiso sin perder el aliento, la apertura, el compromiso y la flexibilidad.

La enseñanza virtual en combinación a la presencial convencional aporta unas ventajas que justifican su rápida expansión, que por su relevancia, se pasan a sintetizar:

- Aumentar la posibilidad de comunicarse e interactuar de modo horizontal y personalizado para conocer, guiar y apoyar a los estudiantes.

- Una fácil actualización de los contenidos, que como el acceso a los cursos se puede realizar, desde cualquier lugar y en cualquier momento; lo mismo en cuanto a las consignas de las actividades y trabajos prácticos parciales y finales.

- Aprovechar las instancias anteriores para llevar adelante no sólo un feed-back de seguimiento inmediato sino enriquecer las formas evaluativas según lo aspirado inicialmente por el programa de formación.

Pero la enseñanza virtual como las propuestas hibridas con presencialidad NO son panaceicas. Los procesos formativos además de ser largos y contradictorios, son dilemáticos. Habrá que superar también mitos como que con la formación mixta se consigue un aprendizaje más autentico y efectivo porque es mas real, o de presentar connotaciones de rapidez y de mayor retención estudiantil.

A pesar de los entusiasmos y de las experiencias -cada vez mayores- de apelar a la integración de enfoques y recursos tecnológicos, no existe aun un solo estudio que demuestre que el aprendizaje sea lo que se enunció si se empleara la formación mixta presencial y en línea en una combinación de métodos.

Si bien se consigue una enseñanza menos improvisada y rutinaria y un aprendizaje más aterrizado y significativo, que reconoce que la retención de un aprendizaje se 
liga a la motivación que el alumno tenga, el sentido que le otorgue a la propuesta formativa, la necesidad que perciba de aprender algo para aplicarlo en su vida cotidiana y/o laboral, los estudios de contratación y de investigación en la acción, siguen siendo centrales, para demostrar que la calidad pedagógica y humana del profesor/a, la adecuación y atractivo de las actividades y materiales de aprendizaje con estrategias dinamizadoras y con todos los recursos cotidianos (desde los más convencionales hasta los más electronificados) para ser apropiados y usados, -a pesar de incertidumbres varias-, siguen siendo insoslayables.

La educación flexible y mixta con todas las tecnologías en el nivel universitario, representa una apuesta de adaptación a las demandas de una alternativa sociedad del conocimiento dada por la incorporación y explotación apropiadas de las TICs a los programas formativos de una manera combinada con la presencialidad, característica de los procesos universitarios convencionales.

Estas sugerencias deberían impulsar en general en las instituciones educativas universitarias, superar situaciones convencionales -muchas de ellas burocratizadas, rigidizadas y alejadas de ser un servicio científico de avanzada para una satisfacción comunitaria-.

La gran presencia de la tecnología y las TICs en las vidas de las personas, grupos y organizaciones sociales exige formación seria y responsable que requieren de diversas decisiones de diferente naturaleza. Todas vinculadas al desarrollo de competencias y dominio para acceder, manejar, integrar, distribuir, evaluar, producir, crear/ recrear y compartir información y así, poder funcionar ágilmente en la sociedad de la información y el conocimiento.

\section{FORMACIÓN CONCEPTUAL Y METODOLÓGICA DEL PROFESORADO UNIVERSITARIO EN SITUACIONES HIBRIDAS O COMBINADAS CON TICS}

La introducción de las TICs en la universidad convencional presencial no se puede llevar a cabo de modo improvisado o aleatorio sino que requiere una planificación estratégica de la organización y una formación del cuerpo de profesores e investigadores muy seria si se desean obtener resultados de calidad. Un punto central en ello es contar también, con una infraestructura tecnológica adecuada para una apropiada enseñanza basada en las tecnologías que involucran a un indiscutido cuerpo de personal de apoyo administrativo, técnico y otros. 
Todos los profesores/as dentro de estas propuestas, se convierten como trabajadores del conocimiento (o e-workers) emergentes de esta sociedad y economía de la información, en analistas simbólicos.

Son líderes en y para la introducción, gerenciamiento, evaluación y prospectiva del cambio educativo, lo que requiere enérgica y continua capacitación y estímulo para protagonizar innovaciones y así, pilotear las TICs (y/o lo que se inventare) para la administración, enseñanza y aprendizaje virtuales, mixtos u otros.

También son o deberían ser gerentes que urgen hacia decisiones y financiamientos versátiles, constantes y sostenidos, que soporten las innovaciones de modo evaluado, a lo largo del tiempo.

El saber y el hacer reflexivo entonces en este contexto caracteriza a los administradores educativos, investigadores/as y profesores y profesoras que como "procesadores/as humanos/as de datos", seleccionan, evalúan, usan y se apropian de la información como materia prima para su desempeño cotidiano en diversas organizaciones formadoras. De dicho reservorio virtual cada vez más enorme y de reproducción interactiva veloz, debe relacionar y extraer, interpretar y elaborar el saber que necesita para transferirlo al contexto educativo y organizacional a través de acciones estratégicas y contrastadas.

Por lo mismo, los profesores/as como analistas simbólicos recurren y demandan constantes propuestas de reciclaje-perfeccionamiento- reformulación de lo que saben, a través de programas de capacitación convencionales y "just in time" con productos y servicios electrónicos (de e/blended learning, videoconferencias y otros) de "self directed profesional self-study inside a lifelong learning" o aprendizaje profesional autodirigido a lo largo de la vida general y profesional.

Concurrente a ello, se presentan algunas propuestas (aunque incluyan incertezas) para entender el tránsito que se propone en organizaciones que se recrean constantemente, tratan de discriminar ventajas e inconvenientes, -ya que panaceas no existen-, y sólo la contrastación con la acción cotidiana lo pondrá en evidencia.

En su trabajo fundamental The Rise of the Networked Society ("El auge de la sociedad interconectada”, 1997), Manuel Castells destaca que «la habilidad (o la falta de habilidad) de las sociedades para controlar la tecnología y en particular las tecnologías que son estratégicamente decisivas en cada época histórica tienen una gran influencia en su destino, hasta el punto de que se podría decir que si bien 
la tecnología per se no determina la evolución histórica y los cambios sociales, sí determina la capacidad de las sociedades para transformarse».

Por lo visto, existe la imperiosa necesidad de formar en nuevas competencias a las personas en general (estudiantes) y a los nuevos agentes (profesores/as e investigadores/as) que trabajan en las organizaciones educativas universitarias, como analistas simbólicos, experimentadores y resolutores de problemas que aplican habilidades de análisis, síntesis y críticas al pensamiento, entre otros rasgos.

Tanto el dominio de las competencias "informacionales" (que se conocen como "information literacy" o "information fluency") como las competencias comunicacionales mediáticas, que se hallan articuladas de modo especial a las habilidades interpersonales de empatía e inteligencia emocional, respeto, solidaridad y colaboración, es lo que debería caracterizar a los agentes de una organización universitaria de genuina vocación de servicio e inclusión social.

Al mismo tiempo se tiende a fortalecer la autodirección y autonomía de los profesores/tutores, que luego transfieren a los estudiantes, con la respectiva capacidad de negociación y adaptación, dentro de un marco de responsabilidad social, aumentan la productividad académica y social.

Sin la formación en estas competencias fundamentales, el profesorado podría dar por veraz o valiosa, alguna información errónea, hacer inferencias e interpretaciones indebidas, pensar que ya sabe lo suficiente y para siempre, dejarse llevar por prejuicios y, en suma, tomar decisiones equivocadas (de las que nadie esta libre mas allá de los grados de capacitación y académicos obtenidos) y poner en marcha, "malas prácticas”, que en los tiempos que corren no es valida a ninguna universidad.

\section{La formación del profesorado y el cambio socio tecnológico}

El cambio socio tecnológico con la articulación crítica de las TICs en la formación del profesorado tiende a revisar y enriquecer la enseñanza convencional presencial. Apunta al conjunto de intervenciones planeadas hacia el logro de saberes tecnológicos ${ }^{13}$, -correspondientes al dominio del hardware, software y mindware (o tecnologías invisibles relacionadas a las estrategias socio-cognitivas que se apoyan en las funciones superiores del pensamiento y en el intercambio con los otros)-, requeridos por toda organización universitaria. 
Algunas sugerencias para la formación del profesorado ${ }^{14}$ universitario presencial en general y que favorezcan el cambio tecnológico en especial, que se propone deberían aprestar y remover mentalidades, es decir, apuntar a lo actitudinal y valórico, lo que es más central que la existencia de artefactos tecnológicos.

Ello se operativiza con una:

- Sensibilización en grupos de entrenamiento que buscan revisar para cambiar el pensamiento del profesor y así, su accionar, por medio de una interacción abierta y sincera.

- Retroalimentación de opiniones realizada de modo formal (a través del uso de cuestionarios) o informal para identificar discrepancias entre las percepciones de los usuarios/estudiantes y superiores; a lo que debe seguir una discusión de conceptos y metodologías, enfoques y estrategias de trabajo pedagógico mediado con tecnologías con una desmistificación de los medios o recursos tecnológicos, en general tomados como panacéicos.

- Consultoría evaluativa durante el proceso a fin de conocer acerca de lo que está pasando e identificar problemas que necesitan reencauzarse para mejorar la calidad de la educación administrada en la combinación presencial y con las TICs.

- Integración de equipos donde la alta interacción entre los miembros (a modo de comunidades de aprendizaje, práctica e investigación) sea su rasgo central lo que incrementará confianza, apertura, productividad y aplicación creativas.

- Desarrollo personal y organizacional para revisar y cambiar las actitudes, erradicar estereotipos y percepciones rígidas que perturban la comunicación y la construcción de conocimiento conjunto, dentro de un clima de respeto y confianza mutua.

Esta propuesta de flexibilización de la enseñanza superior hace que ${ }^{15}$ se acceda e interactué, con evaluación y control activos, a un amplio ábanico de recursos y materiales para el aprendizaje, que deberán ser adaptados y procesados.

Concurrentemente al fortalecer las experiencias personalizadas de aprendizaje, que robustecen la autonomía, se extienden las formas grupales y colaborativas en redes de diverso tipo que desarrollan los valores democráticos, de solidaridad, esfuerzo compartido, de respeto a la diferencia y otros. 
Tratándose de la incorporación de las TICs a una organización universitaria y/o programa educativo superior se percibe a la innovación como una propuesta planificada para generar o mejorar productos, procesos y/o servicios formativos.

Sin embargo, si bien las innovaciones implican cambios, no todos los cambios necesariamente involucran nuevas ideas correspondientes a un know-how que conducen a mejoras socioculturales, educativas y tecnológicas significativas.

Se debe tener claro que aun, en la mayoría de los casos al referirse a las innovaciones en la universidad y/o en la formación del profesorado, se apunta mas a productos, donde el concepto de cambio socio-tecnológico apunta a la incorporación de equipos tecnológicos, y menos a una discusión racional y crítica de las TICs, que abarca el logro de un nuevo know how "invisible". Es decir deberían apuntarse a los procesos de diseño, gestión, producción y seguimiento de las nuevas estructuras o sistemas administrativo-formativos que caracterizan a los programas presenciales que incorporan las TICs. Estas parecen ser centrales -aunque debería evaluarse caso por caso-, en todos los rubros que influyen positivamente en la innovación, facilitan la flexibilidad, la transparencia, la adaptación y la interfertilización por la circulación de la información que cada vez mas compromete al profesor/a, en su rol combinado con la enseñanza presencial.

Claro que -se enfatiza nuevamente-, es necesario que todos estén asociados y comprometidos dentro del marco compartido respecto de las propuestas de innovación mencionadas de flexibilidad organizacional y formativa.

El rol de las redes en la gestión y circulación de la información coadyuva a la transformación en concepción y metodologías de management, de la enseñanza y del aprendizaje, que se realizan tanto dentro como fuera de las paredes de la organización e incluso del aula de formación/capacitación al tratarse de redes virtuales (Harasim y Hiltz, 1999) $)^{16}$.

Esto implica una reformulación de relaciones, en espacios de intenciones sociales diversas. Las personas no se encuentran ahora sólo en el lugar convencional o presencial habitual, sino en el espacio común del ciberespacio. El "aula" para enseñar y aprender es cualquier parte donde se tiene una PC, una línea telefónica o conectividad sin cables, ventana al mundo para aprender a dominar información y construir conocimiento y habilidades varias, vía conexión con la red. Esto implica un gran cambio cultural y de mentalidad como de expectativas sociales. 
Las redes (Harasim y Hiltz, 1999) (op. cit) son grupos de colaboración y producción de saber entre profesores/as como de estudiantes que se comunican y aprenden, trabajan e investigan, de forma conjunta, en el lugar, en el momento y al ritmo que necesiten, del modo más adecuado a su tarea y al solucionar demandas puntuales en términos del acelerado ritmo del cambio socio-tecnológico.

El acento en la participación es digno de retomar dada por la posibilidad tecnológica y en especial por la opción de la Web.2, siempre y cuando, mentalidades e intenciones sociales así lo decidan albergar y sostener ya que sus connotaciones socio-políticas no son nada despreciables.

En consecuencia, existe la necesidad de revisar la cultura institucional universitaria muchas veces, en muchos casos, bastante anacrónica y alejada de una articulación de propuestas más flexibles y dinamizadoras con protagonismo estudiantil y comunitario, con apelación a las TICs, que debería ser tomado en cuenta ya que contribuyen a aumentar su entusiasmo, motivación y retención de los programas formativos.

Se trata de configurar, consolidar y actualizar la cultura organizacional para inscribir proyectos educativos de cambio socio-tecnológico, lo que significa entender, focalizar y optimizar todos los procesos de aprendizaje y enseñanza mediados (y no sólo por tecnología material) dentro de una organización universitaria, o que usar las TICs para "distribuir justo a tiempo", cursos de enseñanza superior a poblaciones cada vez mayores, demandantes de la misma.

Entonces si se desea dar respuesta al interrogante que abrió esta contribución expositiva y reflexiva, se podría sostener que son variados los prerrequisitos necesarios, muchos de los cuales fueron analizados y que darían respuesta de cómo hacer dicho cambio o tránsito. Si la preocupación es como superar barreras para instaurar correctamente los programas de educación universitaria presenciales combinando las TICs e Internet, se podría sostener que es necesario:

- Decididas y sostenibles inversiones tecnológicas de infraestructura y de redes, de una administración, organización y propuestas curriculares de formación adecuada a los protagonistas del programa/currículo que permita la apropiación con uso y aplicación inteligentes, de los recursos materiales y simbólicos, convencionales y electrónicos a variadas situaciones de la vida y el trabajo. 
- Aprender a aprender de un modo autorregulado (metacognitivo que implica fortificar la autonomía de la persona que aprende) en situaciones combinadas presenciales y en línea, o cercanas y virtuales. Se trata primero de reformular de modo científico y práctico el currículo, contrastado en la realidad para que llegue a la situación de enseñanza y aprendizaje a fin de realizar un análisis minucioso de objetivos y contenidos didácticos y optimizar estrategias metodológicas y recursos electrónicos que profundicen el mind y el socioware a través de los potenciales software y hardware presentes en cualquier entorno mediado por tecnología (presencial o remoto).

De este modo, especial dedicación habrá que tener con el estudiante para fortalecer su auto-dirección en el aprendizaje, que de modo autónomo y con apoyo del grupo y del profesor/a presente y en línea, apoyan el interjuego experiencial e instruccional. Ello será posible porque se han adoptado nuevos marcos socio mentales de comunicación y aprendizaje, y nuevas habilidades mediáticas y electrónicas, personales y grupales como asimismo, procesos de lectura interactiva y navegación reflexivas, manejo racional del tiempo para la realización, intercambio y discusión, entrega y defensa de los trabajos prácticos y exámenes finales, si se trata de propuestas de educación formal superior.

La enseñanza virtual en combinación a la presencial convencional aun con los recaudos enunciados, aporta unas ventajas que justifican su rápida expansión, que por su relevancia, se pasan a sintetizar:

- Aumentar la posibilidad de comunicarse e interactuar de modo horizontal y personalizado para conocer, guiar y apoyar a los estudiantes.

- Una fácil actualización de los contenidos, que como el acceso a los cursos se puede realizar, desde cualquier lugar y en cualquier momento; lo mismo en cuanto a las consignas de las actividades y trabajos prácticos parciales y finales.

- Aprovechar las instancias anteriores para llevar adelante no sólo un feed-back de seguimiento inmediato sino enriquecer las formas evaluativas según lo aspirado inicialmente por el programa de formación. 


\section{PARA FINALIZAR}

Se requiere mucha "vigilancia epistemológica" para no reiterar en la educación virtual o en combinación con las TICs, los errores que ha cometido y aún comete la educación presencial convencional pura. Como por ejemplo, caer en el engaño de tratar de reproducir en la red y con las TICs, el perfil de una clase tradicional, sin aprovechar las opciones que brindan estos entornos virtuales en sí mismos al utilizar sus herramientas y características típicas en modalidades que favorezcan el aprendizaje y que son difíciles de hallar para utilizar en la clase tradicional. Sobretodo añadiendo la enorme actualización científica y/o incluyendo puntos de vista contrapuestos para generar debates como también la adaptación de los contenidos a las demandas y los estilos de aprendizaje de los alumnos, de manera de obtener una mayor calidad en la enseñanza, que implícita variados rubros a tener en cuenta.

Lo importante es el contenido en su validezy relevanciajunto con las herramientas (invisibles mentales o del "mindware") cognitivas, sociales, éticas y actitudinales, para profundizar (ya que sólo se usan muy poco de sus potencialidades) para fortalecer el uso valioso de las TICs (con el software y el hardware) y coadyuvar a logros formativos ${ }^{17}$.

Otras variables intervinientes utilizadas para re-orientar la formación superior presencial a través de las TICs e Internet es potenciar lo comunicativo grupal y productivo académico con acciones docentes-tutoriales factuales y virtuales dentro de un proyecto de trabajo grupal que haya reformulado y discernido para qué y porqué elegir la propuesta de combinación de concepciones y metodologías presenciales y electrónicas. Ello puede ser un trabajo de producción de material educativo nuevo, un problema a resolver, un caso a analizar, una investigación a realizar de modo conjunto, etc. lo que implica desmistificar pseudo-expectativas para poner todas las energías en reorganizar en consecuencia, la formación y el estudio de otro modo.

Para que un sistema o programa demuestren ser productivo y renovado o con un desempeño que afiance el cambio tecnológico a través de integrar en los proyectos de aprendizaje las propuestas electrónicas con la presencialidad al interior de una organización universitaria, se piensa que los requerimientos centrales son:

- Superar estadios donde reinan los medios artefactuales per se para transitar hacia el reconocimiento de la centralidad de las mediaciones socioculturales, edu-comunicacionales, organizacionales y tecnológicas desde el punto de vista 
simbólico y no sólo material, a fin de lograr altos niveles de logros en virtud de los procesos e insumos implicados.

- Identificar el desempeño de agentes y sectores al interior de la organización educativa superior en interdependencia porosa con el marco externo, con el despliegue de la evidencia de competencias contrastadas en objetivos que orientan de modo fundamentado, el proceso y las herramientas de aprendizaje seleccionadas con coherencia en la ejecución de los proyectos formativos.

\section{NOTAS}

1 Castoriadis, C. (2006). Una sociedad a la deriva, Edit. Katz Bs Aires.

2 Stake, R.1976. Evaluación respondente.

3 Ciudadanía emergente como diferente respecto a la del siglo XIX: Fainholc, B. "Anticipar la sociedad del conocimiento alternativa con la práctica educativa del socio conectivismo crítico de las TICs para la formación de la ciudadanía”. Ponencia Presentada en la Conferencia Internacional de Educación a Distancia de la UNED de Costa Rica. Nov/2006.

4 Visitar www.secondlife.com

5 La articulación de las TIC en la enseñanza universitaria se halla reconocida, desde hace tiempo, en la ERIC en torno al tópico "Technology in Higher Education", que puede consultarse en la siguiente dirección: http://www.eriche.org/Library/crib/ techinhe.html

6 Fainholc, B. (2001). La Tecnología Educativa Apropiada: una revisita a su campo a comienzos de siglo. Revista RUEDA No, Universidad Nacional de Lujan, $\mathrm{N}^{\circ} 4$, Septiembre. 2001.

7 Bates, T., (2001). “Cómo gestionar el cambio tecnológico”. Cap. 1: Afrontar el reto tecnológico en los centros universitarios. Edit. Gedisa, Barcelona.

8 Baumann, Z. (2004). La sociedad liquida. Edit Paidos. Bs.As.

9 Web.2: Software social que conforma comunidades al democratizar el acceso a herramientas sofisticadas conformando inteligencia colectiva. Pretende reducir la distancia entre los que acceden a la web y los que publican información en ella, de modo gratuito apelando a un gestor de contenidos en forma de blog, pudiendo también, incluir videos (You-tube).

10 Fainholc, B. Ver: www.cediproe.org.ar Redefinicion del rol del Profesor /a en propuestas de aprendizaje mixto (o B-Learning). Experiencia llevada adelante por Cátedra de Tecnología Educativa de UNLP: Prof. Titular Dra Beatriz Fainholc, con el apoyo tecnológico del CTER de la Universidad de Illinois. 2004- 07.

11 Fainholc, B. (2001). La Tecnología Educativa Apropiada: una revisita a su campo a comienzos de siglo. Revista RUEDA No Universidad Nacional de Lujan, $\mathrm{N}^{\circ} 4$, Setiembre. 2001.

12 Senge, P. (1992). La quinta disciplina. Granica. Bs. As.

13 La creación y recreación de saber tecnológico se relaciona con el "conocimiento tácito o implícito”. Es el orientado e inferido desde la acción práctica y reflexiva en cualquier 
área disciplinar y de la vida cotidiana; se adquiere en la experiencia contrastada, con poca o sin ayuda; permite a las personas conseguir objetivos de valor personal; útil para el mundo real; se relaciona con procedimientos; se expresa en condiciones. "si... entonces...". Se vincula a valores sociales y democráticos que buscan mejorar la efectividad organizacional y personal en una contribución al desarrollo social.

14 Fainholc, B. (2000). Formación del profesorado para el nuevo milenio: hacia una Tecnología Educativa Apropiada. Edit Magisterio, Bs. Aires.

15 Salinas, J. (1999). Que se entiende por una institución de educación superior flexible? Comunicación presentada a EDUTEC 99, Sevilla, ISBN: 84-89673-79-9.

16 Harasim, L.; Hiltz, S. y Otros (1999). "Redes de Aprendizaje”. Cap. Las Redes de Aprendizaje: un paradigma para el siglo XXI. Edit. Gedisa, Barcelona.

17 Salomón, G. (1999). La influencia de la tecnología en la mente. Publicación CEDIPROE.

\section{REFERENCIAS BIBLIOGRÁFICAS}

Area Moreira, M. (2004). Los medios y las Tecnologías en la Educación. Madrid: Pirámide.

Bates, T. (2001). Cómo gestionar el cambio tecnológico. Cap. 1: Afrontar el reto tecnológico en los centros universitarios. Barcelona: Gedisa.

Bruner, J. (1996). La educación como puerta de la cultura. Bs. As.: Paidós.

Cabero, J. (2001). Tecnología Educativa. Barcelona: Paidós.

Duart, J.; Sangrá, A. (2000). Aprender en la virtualidad. Barcelona: Gedisa.

Fainholc, B. (2000). La formación del profesor en el nuevo milenio: aportes de la Tecnología Educativa Apropiada. Bs. As: Edit. Magisterio.

Fainholc, B. (2001). La Tecnología Educativa Apropiada: una revisita a su campo a comienzos de siglo. Revista RUEDA. Universidad Nacional de Lujan, $\mathrm{N}^{\mathrm{O}} 4$, Septiembre. 2001

Fainholc, B. (2002). E-learning: utilidad corporativa y desmistificación. Bs. As.: Publicación CEDIPROE.

Fainholc, B. (2003). El concepto de mediación en la Tecnología Educativa Apropiada y Crítica. [en línea] Disponible en: www. cediproe.org.ar [consulta 2007, 26 de octubre].
Fainholc, B. (2006). Knowledge production within practice and inquiry communities through electronic collaborative training and research. International Conference of Open Universities. GUIDE 2006. Rome, $13^{\text {th }}$ and $14^{\text {th }}$ of February 2006

Fainholc, B. (2006). La Tecnología Educativa en crisis. Hacia una conceptualizacion revisitada de su campo. Cátedra de T. E. en UNLP, Bs. As. Argentina.

Ferres, J. (2005). Competencias en la comunicación audiovisual. España: Consejo del Audiovisual de Cataluña.

Gallego Rodriguez, A.; Martínez Caro, E. (2003). Estilos de aprendizaje y elearning: hacia un mayor rendimiento académico. Revista RED, $\mathrm{N}^{\mathrm{o}} 7$ [en línea] Disponible en: http://www.um.es/ead/ red/7/estilos.pdf [consulta 2007, $26 \mathrm{de}$ octubre].

Gimeno Sacristán, J.; Pérez, G. (1992). Comprender y transformar la enseñanza. Madrid: Edit. Morata.

Habermas, J. (1987). Teoría de la acción comunicativa. Madrid: Taurus.

Harasim, L. (1999). Redes de Aprendizaje. Guía para la enseñanza y el aprendizaje en red. Barcelona: Gedisa.

Khan, B. (1997). Web-based training: An introduction (WBI), en: Kkan, B. (edit) 
Web-Based Instruction. Nueva Jersey: Englewood Cliffs.

Lorente, E. (2003). Las Comunidades virtuales de enseñanza-aprendizaje. [en línea] Disponible en: http://www.ucm. es/info/multidoc/multidoc/revista/ num8/eneko.html [consulta 2007, $26 \mathrm{de}$ octubre].

Marqués, P.; Majó, J. (2002). La revolución educativa en la era de Internet. Barcelona: Cisspraxis.
Marqués; P. (2003). Sistemas de teleformación. [en línea] Disponible en: http://dewey.uab.es/pmarques/telefor. htm [consulta 2007, 26 de octubre].

Salomón, G. (1993). Cogniciones distribuídas. Bs. As: Edit. Amorrortu.

Schon, D. (1992). La formación de profesionales reflexivos. Madrid: Paidós. MEC.

Stenhouse, L. (1991). Investigación y desarrollo del curriculum. Madrid: Edit. Morata.

\section{PERFIL ACADÉMICO Y PROFESIONAL DE LA AUTORA}

Beatriz Fainholc es Directora General de la Fundación del CEDIPROE- Centro de Diseño, Producción y Evaluación de Recursos Multimediales para el Aprendizaje. Autora de numerosas colaboraciones en revistas, boletines y portales electrónicos como de libros de la especialidad en una óptica transdisciplinaria aplicada al diseño e implementación de TICs en programas de Tecnología Educativa en la línea y, Educación a Distancia. Evaluadora de proyectos y materiales de E-learning en Foros Nacionales e Internacionales. Investigadora de Ciencia y Tecnología de la UNLP. Miembro consultivo de gabinetes técnicos para proyectos educativos de Tecnología educativa, Educación a Distancia y Tecnologías de la Información y la Comunicación -TICs- en Ministerios y oficinas educativas (nacionales y extranjeras).

E-mail: cedima@ciudad.com.ar

DIRECCIÓN DE LA AUTORA

CEDIPROE- Centro de Diseño, Producción y Evaluación/Investigación de Recursos para el Aprendizaje.

Calle Uruguay 766, P.B. “5” (1015)

Buenos Aires-Argentina

www.cediproe.org.ar

Fechas de recepción del artículo: 17/10/07

Fechas aceptación del artículo: 09/01/08 